Biological samples (blood and cheek-swabs) will be taken from children and parents for obesity and taste gene testing.

Results: The first stage of the follow-up will cover early childhood development from the prenatal period until the age of 3 years. The ongoing analysis will study how mothers weight gain during pregnancy affects child's weight and whether the environmental and genetic influences in development of obesity can be separated. The effect of genetic variation in bitter taste receptor on child feeding, eating patterns and weight gain will also be examined.
Conclusions: The research aims not only at gaining information about the correlations between different factors affecting childhood obesity, but also at producing results having more practical applications for use of childhood obesity prevention in children and families.

Funding: The STEPS study is funded by Academy of Finland, Paivikki and Sakari Sohlberg Foundation, Turku University Foundation, Juho Vainio Foundation and Finnish Cultural Foundation: Varsinais-Suomi Regional Fund.

\title{
20 - Economics of obesity: nutrition and physical activities substitution effect
}

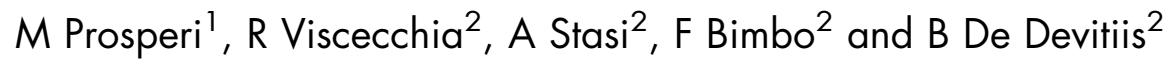 \\ 'University of Foggia, Department of Bioagromed, Foggia, Italy: ${ }^{2}$ Department of PrIME, University of Foggia, Foggia, Italy
}

Introduction: Scientific evidence has demonstrated that child obesity depends on education and economic status of parents, and geographical location. Differently from the majority of published studies, we explain obesity in terms of a rigorous economic model suitable to design a sciencebased health policy scheme. The model is based on a neoclassical approach, and analyses the obesity problem in terms of a set of nutritional and physical activity choices.

Method: We propose a two-step methodology of economic choice modelling. In the first step, we suppose a utility model, where people choose the most efficient combination between calorie intake and energy consumption. In the second step, we estimate the cross-elasticity between the utility of nutrition and that of physical activity, given the observed levels of BMI. A case study referred on a population of 898 students (age 11-19 years) in the South of Italy is presented.
Results: The estimation of the cross-elasticity between and across different types of foods and physical activity, allows the comparison between different social groups. Students belonging to lower income groups show higher preference towards high calorie foods, with respect to healthy food. Similarly, students belonging to parents with higher education, show higher preference towards physical activity, in respect to food consumption.

Conclusions: The estimation of the cross-elasticity is valuable information to public policy aimed at the reduction of obesity. Diversified policy measures may be targeted to specific social groups, in order to increase the effectiveness of the policy and to reduce social inequalities.

Funding: Research relating to this abstract was performed in collaboration with Rotary Club (section of Foggia).

\section{1 - Fluid consumption data extract from a French national survey: INCA2}

\author{
S Vergne ${ }^{1}$, L Le Bellego', F Recours ${ }^{2}$ and $\mathrm{P} \mathrm{Hebel}^{2}$
}

${ }^{1}$ DANONE Research, Palaiseau Cedex, France: ${ }^{2}$ Centre de recherche pour l'étude et l'observation des conditions de vie (CREDOC), Paris, France

Nutritional recommendations are mainly based on dietary surveys of population: this is the case for intake recommendation as well. In order to make consistent recommendation on a European scale, the European Food and Safety Agency
(EFSA) has very recently published a scientific opinion, in which it defines adequate intake of water as $2 \cdot 01 / \mathrm{d}$ for females and $2.5 \mathrm{l} / \mathrm{d}$ for males, from fluids and food moisture. Considering that food moisture contains $20 \%$ of water on 